\title{
Step bunching of vicinal GaN(0001) surfaces during molecular beam epitaxy
}

\author{
M. H. Xie, S. H. Cheung, L. X. Zheng, Y. F. Ng, Huasheng Wu, N. Ohtani,* \\ and S. Y. Tong \\ Department of Physics, The University of Hong Kong, Pokfulam Road, Hong Kong
}

(Received 22 November 1999)

\begin{abstract}
Step bunching of vicinal GaN(0001) surface during epitaxial growth is observed by scanning tunneling microscopy. Large step stiffness and repulsive step-step interaction are suggested based on step morphology observations. The size of the bunch changes with time, depending on the direction in which the substrate is heated by a direct current. This observation provides evidence for the electromigration effect causing the step bunching, and from the field dependence we infer that adatoms, which are likely $\mathrm{N}$, have effective positive charges.
\end{abstract}

Recent interests in $\mathrm{GaN}$ and related materials for blue light and high power device applications have demanded further improvements in the quality of epitaxial thin films. GaN epilayers grown by molecular-beam epitaxy (MBE) on flat heterogeneous substrates (e.g., sapphire, SiC, etc.) have indicated rough surfaces with coarse-grain or granular morphologies or showing mounds due to growth spirals. ${ }^{1-3}$ The search for novel growth strategies and better substrates thus becomes one of the major focuses in this endeavor. One approach, which has been recognized in many other epitaxial systems but has not been fully exploited in GaN growth, is to use vicinal substrates. Recent growth experiments on such substrates have indicated effective suppression of growth spirals and a reduction of threading dislocations. ${ }^{3}$ This may have its origin in growth kinetics. Therefore, the study of microscopic processes of epitaxial growth is of interest from both fundamental and practical points of view.

In a nonequilibrium growth system such as MBE, surface morphology contains important kinetic and dynamic information of the growth. On a vicinal surface, step motion and evolution is usually a result of a delicate balance among multiple microscopic processes; therefore, by monitoring step development, relevant kinetic parameters can be revealed. An example is the observation of step bunching during sublimation of vicinal $\mathrm{Si}(111)$ when heated by a direct current (dc). ${ }^{4,5}$ Subsequent theoretical and experimental investigations of the phenomenon extracted important kinetic details from which the rate-limiting processes and the nature of step-step interaction are determined. ${ }^{5-12}$ On the other hand, step bunching is quite a robust phenomenon, which can be caused by many effects including impurity, strain, the Schwoebel effect, and the aforementioned electromigration. Step bunching also has important implications in device applications of the films, as it may affect the realization of nanoscale quantum structures. It may also be possible to utilize and manipulate step bunches to design novel microstructures for quantum effect devices.

In this paper we present our observation and study of step bunching during $\mathrm{GaN}$ growth on vicinal $\mathrm{SiC}(0001)$ substrates. It is seen that the evolution of steps depends on the direction in which the $\mathrm{dc}$ is applied across the sample. This observation provides evidence for the electromigration effect causing step bunching. From the field dependence, we infer that surface diffusing adatoms possess effective positive charges.

The MBE growth of $\mathrm{GaN}$ was carried out at $650{ }^{\circ} \mathrm{C}$ at a deposition rate of $0.26 \AA / s$. These conditions ensured a stepflow growth mode as indicated by the absence of isolated islands observable by scanning tunneling microscopy (STM). Sample heating was achieved by applying the dc through the sample along the long side of the rectangular substrate (10 $\times 4 \mathrm{~mm}^{2}$ in size). The voltage drop was $8 \mathrm{~V}$ and the current was 0.9 A. The dc direction was deliberately changed for different growth experiments. A N-type 4H-SiC(0001) substrate was nominally $3.5^{\circ}$ off-cut towards [1010] on its $\mathrm{Si}$ face. Following deoxidization at $1100{ }^{\circ} \mathrm{C}$ in a $\mathrm{Si}$ flux, the surfaces showed step and terrace morphologies characteristic of vicinal surfaces. ${ }^{3}$ For GaN growth, the $\mathrm{Ga}$ and $\mathrm{N}$ source fluxes were provided, respectively, from a conventional Knudsen cell at the cell temperature of $980{ }^{\circ} \mathrm{C}$ and from a radio-frequency (rf) plasma generator (Oxford Applied Research, CARS25). The $\mathrm{N}_{2}$ flow rate was $0.13 \mathrm{sccm}$ (standard cubic centimeter per minute) and the rf power was $500 \mathrm{~W}$. The corresponding $\mathrm{Ga} / \mathrm{N}$ flux ratio was measured to be approximately 2 . To quench the growing surface, the dc heating power of the sample was simply switched off. At the same time, the source fluxes were stopped by closing the source shutters as well as by shutting down the rf power of the plasma unit. In situ STM experiments were conducted at room temperature under a constant tunneling current of 0.1 $\mathrm{nA}$ and sample bias of $-3.0 \mathrm{~V}$ (filled state).

Figure 1(a) shows a surface after about $0.5-\mu \mathrm{m} \mathrm{GaN}$ deposition. Regular steps are clearly seen. The height of each step is measured to correspond to double bilayers of $\mathrm{GaN}$, i.e., it equals $c=5.2 \AA$, the height of a unit cell along [0001]. The step edges are straight and align along the [1 $\overline{2} 10]$ direction, normal to the misorientation. The straight steps reflect a high step stiffness of GaN and the regular terrace width suggests an existence of step-step repulsion. ${ }^{13}$ Further growth under the dc bias condition that the electric field points to the step-down direction of the vicinal surface leads to significant step bunching. A surface after $3-\mu \mathrm{m}$ GaN deposition is shown in Fig. 1(b). Comparing to Fig. 1(a), well defined and relatively large bunches of steps have formed. The average 


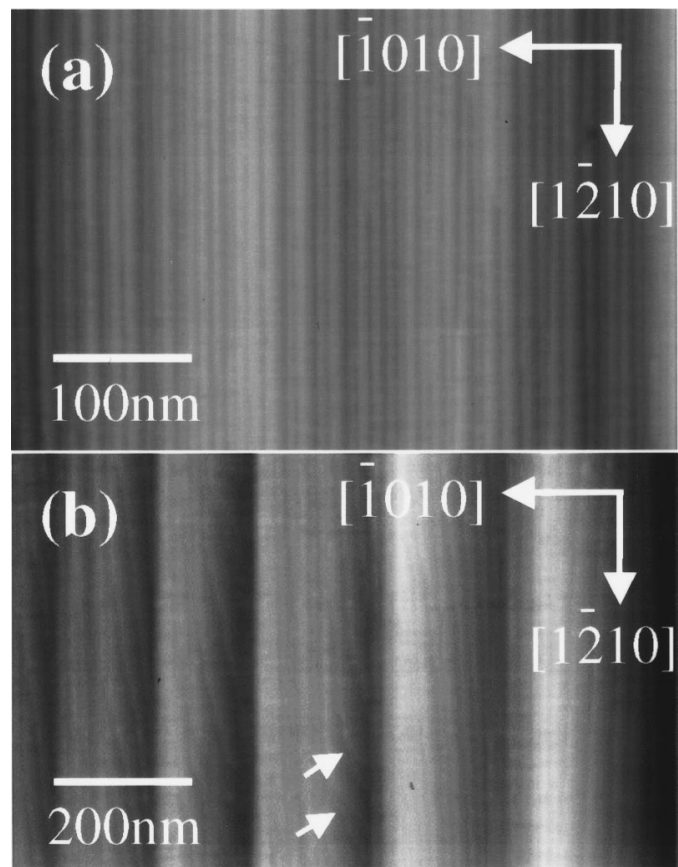

FIG. 1. STM images of GaN surfaces following deposition on vicinal $4 \mathrm{H}-\mathrm{SiC}(0001)$ for (a) $0.5 \mu \mathrm{m}$ and (b) $3 \mu \mathrm{m}$, respectively. The dc bias of the sample during growth is along the step-down direction of the vicinal surface, which is from the right to the left of the image.

separation between the step bunches is about $2000 \AA$ and the height is approximately $100 \AA$. The latter corresponds to $\sim 20$ double bilayers. Continued growth, but under a reverse dc bias direction leads to gradual dissolving of the bunches, i.e., it debunches.

From the image of Fig. 1(b), a few additional features are revealed. The first feature is that the steps in a bunch do not form a macrostep, rather they are separated by small distances. This once again suggests the existence of a repulsive step-step interaction. ${ }^{13}$ The second feature is the presence of crossing steps that connect two neighboring bunches. This agrees with the picture that a step bunch evolves by individual steps capture from the top and release from the bottom of the bunch. ${ }^{6,7}$ Step height measurements of the crossing steps as well as those within the bunch show that they are all double bilayers (i.e., $5.2 \AA$ ), except for a few crossing steps indicated by arrows in Fig. 1(b) which are single bilayer high. These single bilayer height steps are aligned $60^{\circ}$ with respect to the [1010] direction. The formation of double bilayer steps on $\mathrm{GaN}$ has been documented as due to strong anisotropy in the growth rate between two consecutive single bilayer steps, which belong to type- $A$ and type- $B$, respectively. ${ }^{14}$ Upon turning $60^{\circ}$, a step edge changes character from type- $A$ to type- $B$, or vice versa. Therefore, single bilayer height crossing steps are observed when they align $60^{\circ}$ away from [ $\left.\overline{1} 010\right]$. In order to maintain the double bilayer step structure during growth, the speed of growth of the slow-moving type- $B$ step has to be attachment-rate limited. Otherwise, if it were diffusion-rate limited, the speed of advance of the fast-moving type- $A$ step would also be diffusion limited. In this case, the anisotropy described above causing double bilayer step formation would no longer manifest itself unless diffusion itself is anisotropic. The latter has been

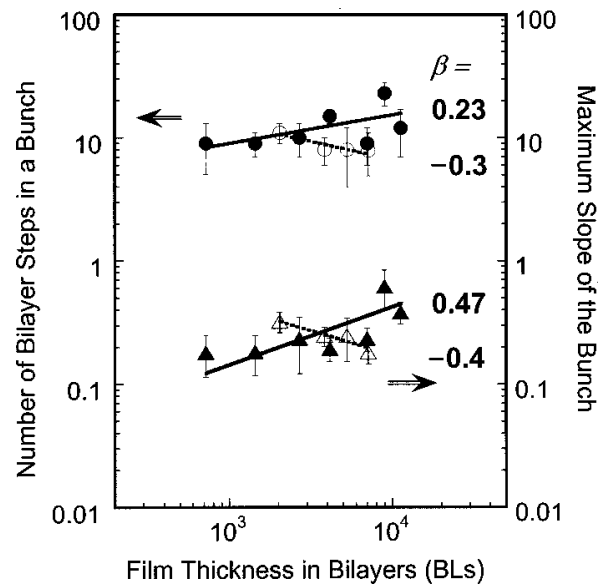

FIG. 2. Film thickness (growth time) dependence of the bunch heights (open and filled circles) and slopes (open and filled triangles). The filled symbols represent data when the dc is along the step-down direction, and the open symbols show data when the bias is along the step-up direction. The lines are least-square fits to the data using the scaling relationship $\sim t^{\beta}$, and the determined values of the exponent $\beta$ are indicated in the figure against each fitted line.

shown not to be a factor in $\mathrm{GaN}$ growth under the conditions used for this experiment. ${ }^{14}$ Consequently, it can be concluded that the rate of growth is limited by adatom attachment at type- $B$ steps.

As mentioned earlier, there are multiple causes that can lead to surface instability towards step bunching. However, for $\mathrm{GaN}$, the dependence of the bunching behavior on the dc direction points to the electromigration effect. The phenomenon is interpreted as caused by an asymmetry of adatom diffusion along step-up and step-down directions due to an external force, $F=Z^{*} e E$, exerted on surface adatoms. Here $E$ is the electric field and $Z^{*}$ is the effective charge of adatoms measured in unit of electron charge $e .^{8}$ In general, if there is a net diffusion in the step-down direction, steps will be unstable and tend to form bunches during growth (the reverse is true for sublimation). Step bunching of $\mathrm{GaN}$ when the electric field is along the step-down direction thus suggests effective positive charges of the surface diffusing species. Under the excess-Ga condition of the growth, $\mathrm{N}$ adatoms are likely the relevant diffusion species. ${ }^{15,16}$ Therefore, based on the above reasoning, $\mathrm{N}$ adatoms possess effective positive charges. Even though the origin of effective charges of surface adatoms can be quite complex, ${ }^{9}$ the positively charged $\mathrm{N}$ adatoms are consistent with the fact that reactive $\mathrm{N}$ atoms from a plasma source include positive ions. ${ }^{17}$

Figure 2 summarizes the film thickness (growth time) dependence of the overall height (or equivalently the number of individual steps in a bunch) as well as the slope of the bunch. In the figure, results under both dc bias conditions, namely along the step-up and step-down directions, are given and each data is an average of more than 20 measurements. The error bars represent the standard deviation from the mean. The lines are the least-square fits to the data using the scaling relationship $\sim t^{\beta}{ }^{10-12}$ Because of the relatively large errors and scatter in the data, one cannot be too certain of the fitted values of the exponent $\beta$. Nevertheless, of the four values of $\beta$ determined in this way, the result $\beta \sim 0.23$ from the bunch height data when the electric field points in the step-down 
direction is close to the value of 0.25 derived from the onedimensional continuum model, ${ }^{11}$ but it does not agree with the two-dimensional model or that of $\mathrm{Si}$ sublimation (in which case, $\beta \sim 0.5$ was obtained). ${ }^{5}$ This suggests there is little lateral fluctuation of the steps during growth, which is consistent with the observed straight steps. Similarly, reversing the dc bias causes the steps to debunch, the slope decays with $\beta \sim-0.4$. This is close to the value of 0.5 that is derived from a model when considering local adatom conservation, ${ }^{10}$ i.e., the mass transport is confined on a given terrace and exchanges of adatoms between terraces are forbidden. ${ }^{12}$ From the same model, if steps are "transparent," an exponent of $\beta=-1$ will result which disagrees with the data.

To summarize, step bunching of GaN during MBE growth is observed. The electric-field dependence of the bunching behavior suggests the effect of electromigration. Moreover, effective positive charges of surface diffusing adatoms are inferred. The dynamic behavior of the bunching process indicates a step growth kinetics that can be described by a one-dimensional kinetic model, where steps are considered nontransparent and the rate of growth is attachment limited.

The authors benefited from discussions with Dr. M. Altman. This work was supported in part by HK RGC Grants Nos. HKU7118/98P, 7142/99P, 7117/98P, 260/95P, HKU CRCG Grant No. 10201825, US DOE Grant No. DE-FG0284ER 45076, and NSF Grant No. DMR-9214054.
*Also at Nippon Steel Corporation, Advanced Technology Research Laboratory, 5-10-1 Fuchinobe, Sagamihara 229-8551, Japan.

${ }^{1}$ E. J. Tarsa, B. Heying, X. H. Wu, P. Fini, S. P. DenBaars, and J. S. Speck, J. Appl. Phys. 82, 5472 (1997).

${ }^{2}$ A. R. Smith, V. Ramachandran, R. M. Feenstra, D. W. Greve, M.-S. Shin, M. Skowronski, J. Neugebauer, and J. E. Northrup, J. Vac. Sci. Technol. A 16, 1641 (1998).

${ }^{3}$ M. H. Xie, L. X. Zheng, Y. F. Ng, Huasheng Wu, N. Ohtani, and S. Y. Tong (unpublished).

${ }^{4}$ A. V. Latyshev, A. L. Aseev, A. B. Krasilnikov, and S. I. Stenin, Surf. Sci. 213, 157 (1989).

${ }^{5}$ Y.-N. Yang, E. S. Fu, and E. D. Williams, Surf. Sci. 356, 101 (1996).

${ }^{6}$ D. Kandel and J. D. Weeks, Phys. Rev. Lett. 74, 3632 (1995).

${ }^{7}$ D.-J. Liu, J. D. Weeks, and D. Kandel, Surf. Rev. Lett. 4, 107 (1997).

${ }^{8}$ S. Stoyanov, Jpn. J. Appl. Phys., Part 1 30, 1 (1991).
${ }^{9}$ D. Kandel and E. Kaxiras, Phys. Rev. Lett. 76, 1114 (1996).

${ }^{10}$ S. Stoyanov, Surf. Sci. 416, 200 (1998).

${ }^{11}$ H. Dobbs and J. Krug, J. Phys. I 6, 413 (1996).

${ }^{12}$ D.-J. Liu, E. S. Fu, M. D. Johnson, J. D. Weeks, and E. D. Williams, J. Vac. Sci. Technol. B 14, 2799 (1996).

${ }^{13}$ E. D. Williams and N. C. Bartelt, in Handbook of Surface Science, edited by W. N. Unertl, Physical Structure Vol. 1 (Elsevier, New York, 1996), Chap. 2, p. 51.

${ }^{14}$ M. H. Xie, S. M. Seutter, W. K. Zhu, L. X. Zheng, Huasheng Wu, and S. Y. Tong, Phys. Rev. Lett. 82, 2749 (1999).

${ }^{15}$ T. Zywietz, J. Neugebauer, and M. Scheffler, Appl. Phys. Lett. 73, 487 (1998).

${ }^{16}$ N. Newman, J. Ross, and M. Rubin, Appl. Phys. Lett. 62, 1242 (1993).

${ }^{17}$ W. C. Hughes, W. H. Rowland, Jr., M. A. L. Johnson, S. Fujita, J. W. Cook, Jr., J. Ren, and J. A. Edmond, J. Vac. Sci. Technol. B 13, 1571 (1995). 